\title{
Perturbation-Iteration Algorithm to Solve Fractional Giving Up Smoking Mathematical Model
}

\author{
M. Khalid \\ Department of Mathematical Sciences \\ Federal Urdu University of Arts, Sciences \& Techonology \\ University Road, Karachi-75300, Pakistan
}

\author{
Fareeha Sami Khan \\ Department of Mathematical Sciences \\ Federal Urdu University of Arts, Sciences \& Technology \\ University Road, Karachi-75300, Pakistan
}

\author{
Azhar Iqbal \\ Department of Basic Sciences \\ Dawood University of Engineering \& Technology, \\ M.A Jinnah Road, Karachi-74800, Pakistan
}

\begin{abstract}
In this paper, a numerical technique is applied to a five variable giving up smoking fractional mathematical model. This model is based on five types of smokers, i.e. potential, occasional, heavy, temporary quitters and permanent quitters. Efficacy of Perturbation Iteration Algorithm on fractional system of differential equations is shown graphically between standard Runge-Kutta method and PIA.
\end{abstract}

\section{Keywords}

Smoking, Perturbation Iteration Algorithm, Caputo Fractional Derivative, Stability Analysis

\section{INTRODUCTION}

Tobacco smoking is the main source of preventable death and is assessed to kill more than 5 million individuals worldwide every year and this number is relied upon to develop. By world health organization report on the worldwide tobacco scourge, use of tobacco, murders or kills numerous individuals in their most beneficial years. Smokers who dont die somehow are caught in serious diseases such as asthma, lung cancers, heart disease, mouth ulcers, etc. [1]

Since last many decades, biological mathematical models of integer order are being studied very successfully through numerical methods. But complex fractional mathematical models are major challenge for researchers. The analysis of the conduction of fractional mathematical models requires the in depth learning. Examples of some physical phenomenon maybe like; heat flow, wave propagation into the shallow waters and the equations governing shock wave phenomenon [2-4]. Similarly in biomathematics it includes HIV, Dengue Fever, Lake Pollution, Biological Population and Giving up Smoking Model etc [5-7]. Zaman et al. discussed smoking dynamics of integer order in detail [8-12]. Also Zeb et al. analyzed it by Homotopy Analysis Method [13].
Stability analysis of Giving up Smoking model has been discussed by most researchers but its fractional order mathematical model has yet not been considered by many. Therefore in this work a very reliable and efficient method is being provided which gives its numerical solutions for all five kinds of smokers and discuss their behavior.

\section{BASIC DEFINITIONS OF FRACTIONAL CALCULUS}

Most normally utilized definitions of fractional derivatives and integrals are Riemann-Liouville and Caputo sense, so here is a brief introduction to these concepts.

\subsection{Definition}

The Riemann-Liouville fractional integral operator of order $\alpha>0$, of a function $f \in \mathbb{C}_{\mu}, \mu>-1$, is defined as

$$
J^{\alpha} y(t)=\frac{1}{\Gamma(\alpha)} \int_{0}^{t}(t-s)^{\alpha-1} y(s) d s ; \quad \alpha>0, t>0
$$

Some properties of the operator $J^{\alpha}$, for $y \in \mathbb{C}_{\mu}, \mu \geq-1, \alpha, \beta \geq 0$ and $\gamma \geq-1$, used in this text are:

(1) $J^{\alpha} J^{\beta} y(t)=J^{\alpha+\beta} y(t)$

(2) $J^{\alpha} t^{\gamma}=\frac{\Gamma(\gamma+1)}{\Gamma(\gamma+\alpha+1)} t^{\gamma+\alpha}$

\subsection{Definition}

A real function $y(x), x>0$ is said to be in space $\mathbb{C}_{\mu}, \mu \in \mathbb{R}$ if there exists a real number $p>\mu$, such that $y(t)=t^{p} y_{1}(t)$, where $y_{1}(t) \in \mathbb{C}(0, \infty)$, and it is said to be in the space $\mathbb{C}_{\mu}^{n}$ if and only if $y^{n} \in \mathbb{C}_{\mu}, n \in \mathbb{N}$. 


\subsection{Definition}

The fractional derivative of $y(t)$ in the Caputo sense is defined as

(1) $D^{\alpha} y(t)=\frac{d^{m}}{d t^{m}}\left(J^{m-\alpha} y(t)\right)$

(2) $D^{\alpha} y(t)=J^{m-\alpha}\left(\frac{d^{m}}{d t^{m}} y(t)\right)$

For $m-1<\alpha \leq m, m \in N, t>0$ and $y \in \mathbb{C}_{-1}^{m}$.

Firstly, Caputo fractional derivative evaluates only an ordinary derivative, then through fractional integral obtains the required fractional derivative. This approach resembles very much like Riemann-liouville fractional integral operator and the integer order integration are linear integration:

$$
J^{\alpha}\left(\sum_{i=1}^{n} c_{i} y_{i}(t)\right)=\sum_{i=1}^{n} c_{i} J^{\alpha} y_{i}(t)
$$

where $\left\{c_{i}\right\}_{i=1}^{n}$ are constants.

\section{PERTURBATION ITERATION ALGORITHM (PIA) FOR FRACTIONAL ORDER}

Consider the following system of fractional order differential equations such as

$$
F_{k}\left(D^{\alpha} y_{k}, y_{j}, t\right)=0 ; 0<\alpha \leq 1, t>0, y \in \mathbb{R}
$$

with initial condition $\frac{d^{\alpha}}{d t^{\alpha}} y(t, 0)=y_{k}(t) ; k=0,1,2, \ldots, m-1$. Introducing $\epsilon$ with nonlinear and fractional derivative term yield

$$
F_{k}\left(D^{\alpha} y_{k}, y_{j}, \epsilon, t\right)=0 ; k=1,2,3, \ldots, K, j=1,2,3, \ldots, K
$$

where $K$ is a representative of the number of differential equations in the system and the number of dependent variables. In this work only $P I A(1,1)$ is considered by taking only one correction term in the perturbation expansion i.e. $n=1, m=1$. The fractional order system of differential equation becomes

$$
\begin{aligned}
F_{1}= & F_{1}\left(D^{\alpha} y_{1}, y_{1}, y_{2}, y_{3}, \ldots, y_{K}, \epsilon, t\right)=0 \\
F_{2}= & F_{2}\left(D^{\alpha} y_{2}, y_{1}, y_{2}, y_{3}, \ldots, y_{K}, \epsilon, t\right)=0 \\
F_{3}= & F_{3}\left(D^{\alpha} y_{3}, y_{1}, y_{2}, y_{3}, \ldots, y_{K}, \epsilon, t\right)=0 \\
& \cdot \\
& \cdot \\
& \cdot \\
F_{k}= & F_{k}\left(D^{\alpha} y_{K}, y_{1}, y_{2}, y_{3}, \ldots, y_{K}, \epsilon, t\right)=0
\end{aligned}
$$

Only $n$ correction terms in this perturbation expansion will be acknowledged

$$
y_{k, n+1}=y_{k, n}+\epsilon y_{k, n}^{c}
$$

where $\epsilon$ is the perturbation parameter. The developed perturbation iteration algorithm is given here as $\operatorname{PIA}(n, m)$; here $n$ are the terms involved in the expansion, $m$ is the $m^{\text {th }}$ order derivative in the Taylor Series expansion provided $n \leq m$ with respect to $\epsilon$, then comparing the coefficient of same power of $\epsilon$ renders the unknown correction terms. Back substitution of these results in Eq.(3) thus yield an algorithm for solution of Eq.(1). Substituting Eq.(4) in
Eq.(3), expanding in a Taylor Series with first derivative only yield.

$$
\begin{aligned}
& F_{K}\left(D^{\alpha} y_{k}, y_{j}, 0, t\right)+\left(F_{K}\right)_{D^{\alpha} y_{k}}\left(D^{\alpha} y_{k}, y_{j}, 0, t\right) \epsilon\left(D^{\alpha} y_{k}\right)_{c}+ \\
& \left(F_{K}\right)_{\epsilon}\left(D^{\alpha} y_{k}, y_{j}, 0, t\right) \epsilon=0
\end{aligned}
$$

where all derivatives are evaluated at $\epsilon=0$. Starting with the initial condition $y_{\circ}(t)$ first $\left(y_{\circ}\right)_{c}$ has been calculated by the help of Eq.(5). Then we substitute $\left(y_{\circ}\right)_{c}$ into Eq.(4) to find $y_{1}$. Iteration process is repeated using Eq.(4) and Eq.(5) until we obtain a satisfactory result.

\section{GIVING UP SMOKING MODEL}

Mathematical model for giving up smoking model is discussed in detail for integer order in [1].

Consider $T(t)$ be the total population with respect to time. Then the five types of smokers are denoted by potential smokers $P(t)$, occasional smokers $L(t)$, heavy smokers $S(t)$, temporary quitters $Q(t)$ and smokers who quit permanently $R(t)$. where $T(t)=P(t)+L(t)+S(t)+Q(t)+R(t)$. For the first time, this model was discussed for four non-linear differential equations but to make it more accurate this model was reconsidered in [1] by weighing the importance of occasional and heavy smokers as two different subclasses, therefore the following system of five different differential equations is considered by assuming that $b$ is the contact rate between potential and occasional smokers, and $c$ is the contact rate between occasional and heavy smokers. Whereas $a$ is the rate of natural death, $f$ is the contact rate between smokers and temporary quitters who revert back to smoking, $\mathrm{d}$ is the rate of quitting smoking, $(1-e)$ is the fraction of smokers who temporarily quit smoking (at $a$ rate $d$ ), $e$ is the remaining fraction of smokers who permanently quit smoking (at $a$ rate $d$ ) [14].

$$
\begin{gathered}
\frac{d P}{d t}=a(1-P)-b P S \\
\frac{d L}{d t}=-a L+b P L-c L S \\
\frac{d S}{d t}=-(a+d) S+c L S+f Q \\
\frac{d Q}{d t}=-(a+f) Q+d(1-e) S \\
\frac{d R}{d t}=-a R+e d S
\end{gathered}
$$

\section{APPLICATION OF PERTURBATION ITERATION ALGORITHM}

Fractional order system of Eqs.(6a - 6e) becomes for $0<\alpha \leq 1$

$$
\begin{gathered}
D^{\alpha} P=a(1-P)-b P S \\
D^{\alpha} L=-a L+b P L-c L S \\
D^{\alpha} S=-(a+d) S+c L S+f Q \\
D^{\alpha} Q=-(a+f) Q+d(1-e) S \\
D^{\alpha} R=-a R+e d S
\end{gathered}
$$

Here $F_{1}$ is $P(t), F_{2}$ is $L(t), F_{3}$ is $S(t), F_{4}$ is $Q(t)$ and $F_{5}$ is $R(t)$ as described in Eqs. (7a - 7e). On applying Perturbation Iteration Algorithm on Eq. (3) following corrected equations have been 


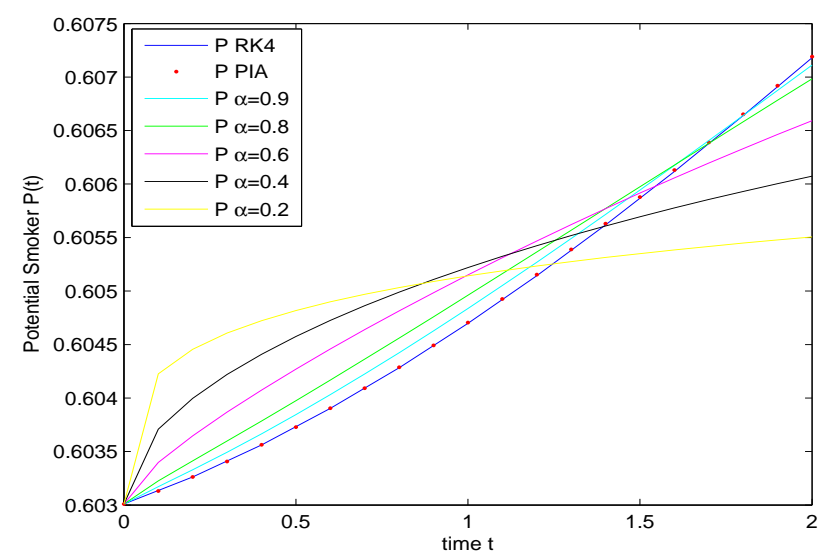

Fig. 1. Comparison of RK4 and PIA along with different values of $\alpha$ for $\mathrm{P}(\mathrm{t})$

obtained

$$
\begin{gathered}
P^{c}(t)=J^{\alpha}\left(-D^{\alpha} P(t)+(1-a) P(t)-b P(t) S(t)\right) \\
L^{c}(t)=J^{\alpha}\left(-D^{\alpha} L(t)-a L(t)+b P(t) L(t)-c L(t) S(t)\right) \\
S^{c}(t)=J^{\alpha}\left(-D^{\alpha} S(t)-(a+d) S(t)+c L(t) S(t)+f Q(t)\right) \\
Q^{c}(t)=J^{\alpha}\left(-D^{\alpha} Q(t)-(a+f) Q(t)+d(1-e) S(t)\right) \\
R^{c}(t)=J^{\alpha}\left(-D^{\alpha} R(t)-a R(t)+e d S(t)\right)
\end{gathered}
$$

Four different set of initial values are considered to verify the efficiency and accuracy of this method. Parameters have same values for all these sets i.e. $a=0.04, b=0.23, c=0.3, d=0.2$, $e=0.4, f=0.25$

\subsection{Case I}

Considered set of initial values is $P(0)=0.60301, L(0)=$ $0.24000, S(0)=0.10628, Q(0)=0.03260, R(0)=0.01811$. Iterations for Perturbation iteration method for first set of initial values are given below.

Iteration for Potential Smokers $P(t)$ :

$$
\begin{aligned}
P_{\circ}= & 0.60301 \\
P_{1}= & 0.60301+\frac{0.0011393823559999947 x^{\alpha}}{\Gamma(1+\alpha)} \\
P_{2}= & 0.60301+\frac{0.0011393823559999947 x^{\alpha}}{\Gamma(1+\alpha)}+ \\
& \frac{0.001272587506888994 x^{2 \alpha}}{(1+2 \alpha)}+\ldots
\end{aligned}
$$

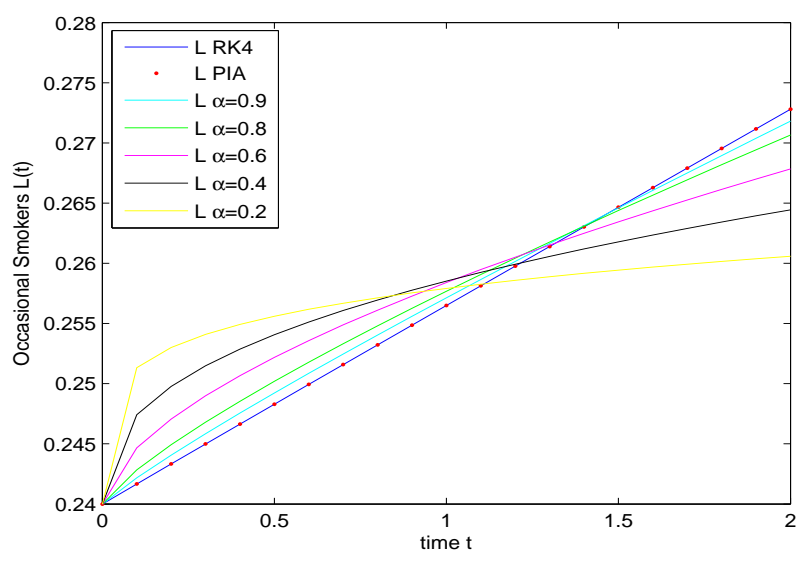

Fig. 2. Comparison of RK4 and PIA along with different values of $\alpha$ for $\mathrm{L}(\mathrm{t})$

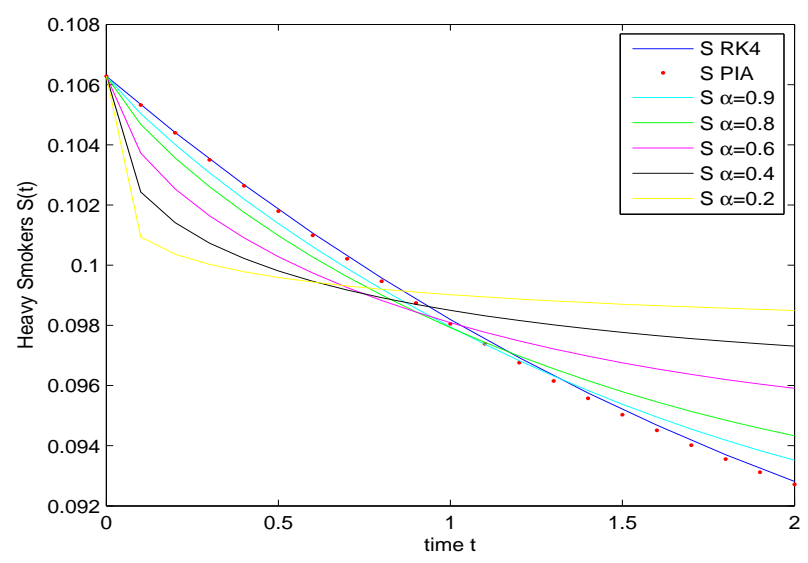

Fig. 3. Comparison of RK4 and PIA along with different values of $\alpha$ for $\mathrm{S}(\mathrm{t})$

Iterations for Occasional Smoker $L(t)$ :

$$
\begin{aligned}
L_{\circ}= & 0.24 \\
L_{1}= & 0.24-\frac{0.0025119423559999956 x^{\alpha}}{\Gamma(1+\alpha)} \\
L_{2}= & 0.24-\frac{0.0025119423559999956 x^{\alpha}}{\Gamma(1+\alpha)}- \\
& \frac{0.0004388314568102905 x^{2 \alpha}}{(1+2 \alpha)}+\ldots
\end{aligned}
$$




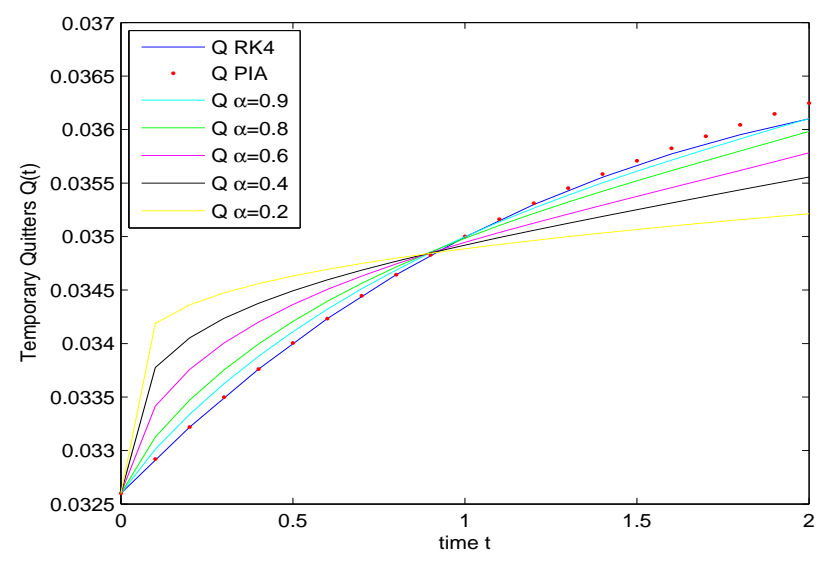

Fig. 4. Comparison of RK4 and PIA along with different values of $\alpha$ for $\mathrm{Q}(\mathrm{t})$

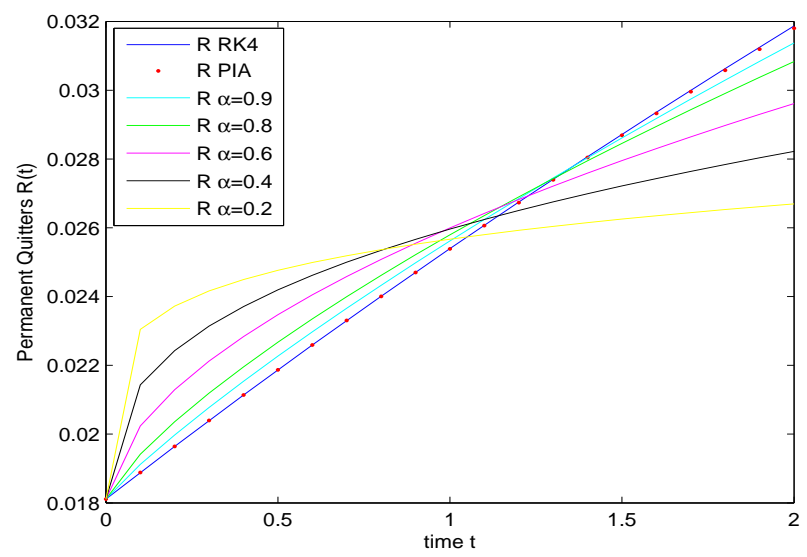

Fig. 5. Comparison of RK4 and PIA along with different values of $\alpha$ for $\mathrm{R}(\mathrm{t})$

Iterations for Heavy Smokers $S(t)$ :

$$
\begin{aligned}
S_{\circ}= & 0.10628 \\
S_{1}= & 0.10628-\frac{0.00970504000000002 x^{\alpha}}{\Gamma(1+\alpha)} \\
S_{2}= & 0.10628+\frac{0.0025119423559999956 x^{\alpha}}{\Gamma(1+\alpha)}+ \\
& \frac{0.0004388314568102905 x^{2 \alpha}}{(1+2 \alpha)}+\ldots
\end{aligned}
$$

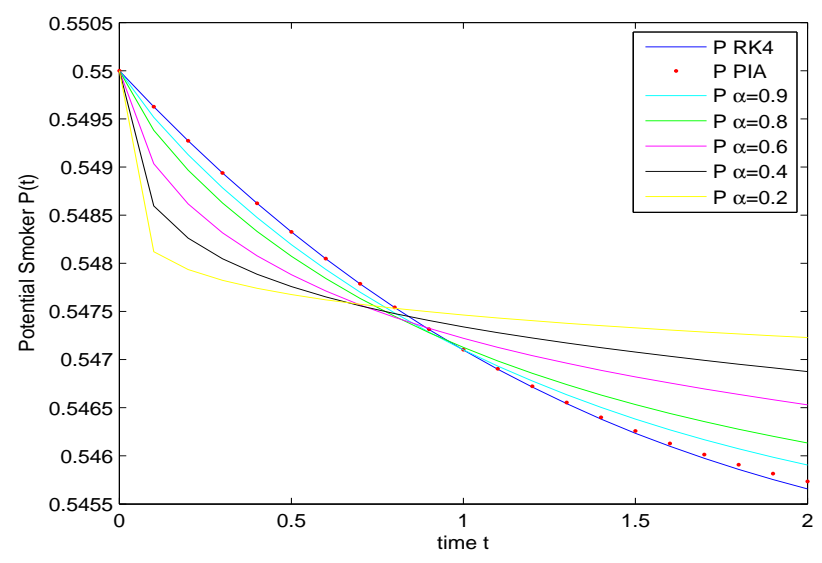

Fig. 6. Comparison of RK4 and PIA along with different values of $\alpha$ for $\mathrm{P}(\mathrm{t})$

Iterations for Temporary Quitters $Q(t)$ :

$$
\begin{aligned}
Q_{\circ}= & 0.0326 \\
Q_{1}= & 0.0326+\frac{0.003299599999999998 x^{\alpha}}{\Gamma(1+\alpha)} \\
Q_{2}= & 0.0326+\frac{0.003299599999999998 x^{\alpha}}{\Gamma(1+\alpha)}- \\
& \frac{0.0021214887999999998 x^{2 \alpha}}{(1+2 \alpha)}+\ldots
\end{aligned}
$$

Iterations for Permanent Quitters $R(t)$ :

$$
\begin{aligned}
R_{\circ}= & 0.01811 \\
R_{1}= & 0.01811+\frac{0.007778000000000002 x^{\alpha}}{\Gamma(1+\alpha)} \\
R_{2}= & 0.01811+\frac{0.007778000000000002 x^{\alpha}}{\Gamma(1+\alpha)}- \\
& \frac{0.0010875232000000002 x^{2 \alpha}}{(1+2 \alpha)}+\ldots
\end{aligned}
$$

Fig 1-5 shows the accuracy of Perturbation Iteration Method. Also, different values of $\alpha$ show the time-fractional behavior of smokers. In this set of initial value we can clearly see the impact of smoking on heavy smokers. These figures represent the extinction behavior of heavy smokers if not discontinued. All calculations are done with the help of Mathematica 9.0.

\subsection{Case II}

Considered set of initial values is $P(0)=0.55000, L(0)=$ $0.20000, S(0)=0.17272, Q(0)=0.06700, R(0)=0.01028$. Iterations for Perturbation iteration method for second set of initial values are given below.

Iteration for Potential Smokers $P(t)$ : 


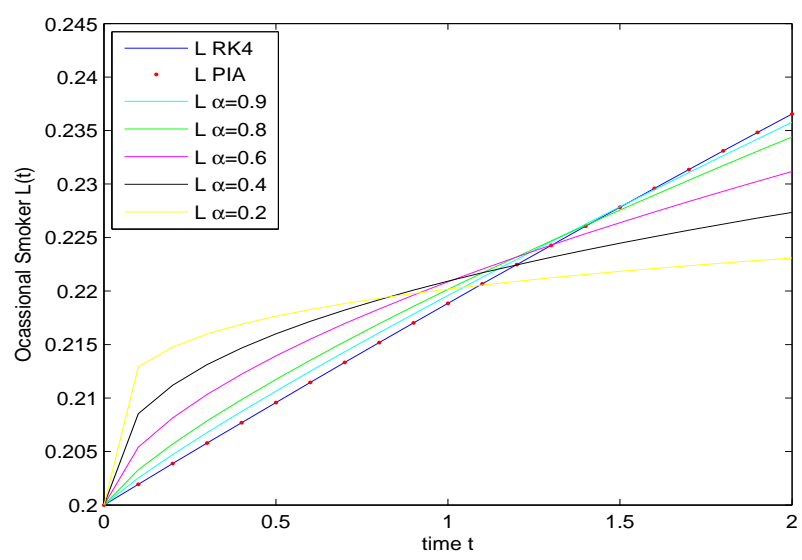

Fig. 7. Comparison of RK4 and PIA along with different values of $\alpha$ for $\mathrm{L}(\mathrm{t})$

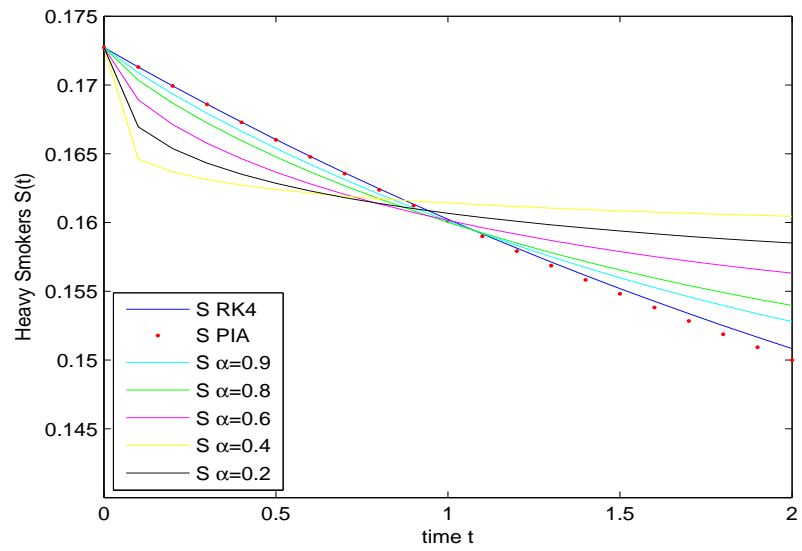

Fig. 8. Comparison of RK4 and PIA along with different values of $\alpha$ for $S(\mathrm{t})$

$$
\begin{aligned}
P_{\circ}= & 0.55000 \\
P_{1}= & 0.55000+\frac{0.00384490800000000117 x^{\alpha}}{\Gamma(1+\alpha)} \\
P_{2}= & 0.55000+\frac{0.00384490800000000117 x^{\alpha}}{\Gamma(1+\alpha)}+ \\
& \frac{0.0021208296124480015 x^{2 \alpha}}{(1+2 \alpha)}+\ldots
\end{aligned}
$$

Iterations for Occasional Smoker $L(t)$ :

$$
\begin{aligned}
L_{\circ}= & 0.20000 \\
L_{1}= & 0.20000+\frac{0.003485880000000007 x^{\alpha}}{\Gamma(1+\alpha)} \\
L_{2}= & 0.20000+\frac{0.003485880000000007 x^{\alpha}}{\Gamma(1+\alpha)}- \\
& \frac{0.0014265499705280015 x^{2 \alpha}}{(1+2 \alpha)}+\ldots
\end{aligned}
$$

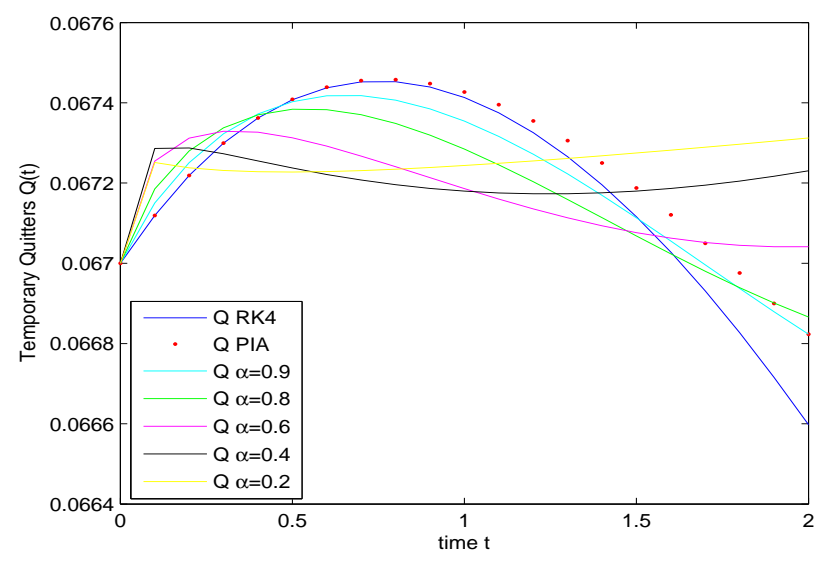

Fig. 9. Comparison of RK4 and PIA along with different values of $\alpha$ for $\mathrm{Q}(\mathrm{t})$

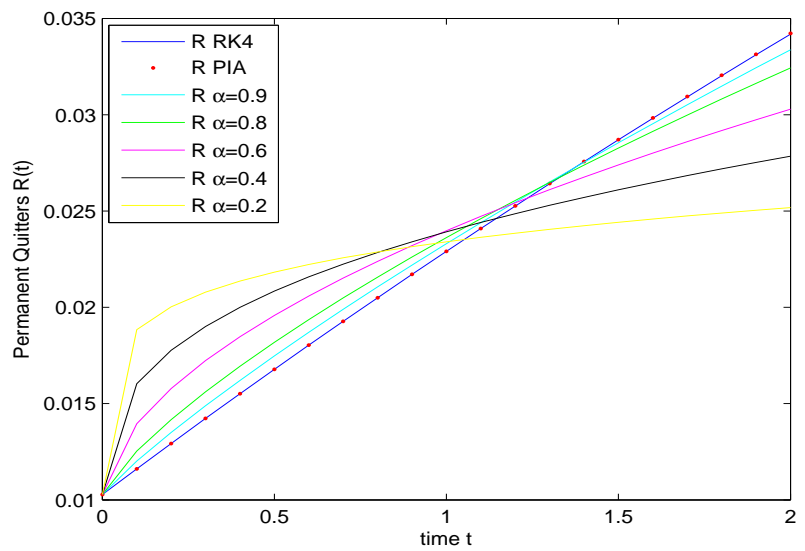

Fig. 10. Comparison of RK4 and PIA along with different values of $\alpha$ for $\mathrm{R}(\mathrm{t})$

Iterations for Heavy Smokers $S(t)$ :

$$
\begin{aligned}
S_{\circ}= & 0.17272 \\
S_{1}= & 0.17272-\frac{0.014339600000000001 x^{\alpha}}{\Gamma(1+\alpha)} \\
S_{2}= & 0.17272-\frac{0.014339600000000001 x^{\alpha}}{\Gamma(1+\alpha)}+ \\
& \frac{0.0030858523580800006 x^{2 \alpha}}{(1+2 \alpha)}+\ldots
\end{aligned}
$$


Iterations for Temporary Quitters $Q(t)$ :

$$
\begin{aligned}
Q_{\circ}= & 0.067 \\
Q_{1}= & 0.067+\frac{0.0012963999999999996 x^{\alpha}}{\Gamma(1+\alpha)} \\
Q_{2}= & 0.067+\frac{0.0012963999999999996 x^{\alpha}}{\Gamma(1+\alpha)}- \\
& \frac{0.002096708 x^{2 \alpha}}{(1+2 \alpha)}+\ldots
\end{aligned}
$$

Iterations for Permanent Quitters $R(t)$ :

$$
\begin{aligned}
R_{\circ}= & 0.01028 \\
R_{1}= & 0.01028+\frac{0.013406400000000004 x^{\alpha}}{\Gamma(1+\alpha)} \\
R_{2}= & 0.01028+\frac{0.013406400000000004 x^{\alpha}}{\Gamma(1+\alpha)}- \\
& \frac{0.0016834240000000004 x^{2 \alpha}}{(1+2 \alpha)}+\ldots
\end{aligned}
$$

In Figure 6-10 the dangerous behavior of smokers towards extinction is shown. If smokers continued the same amount of tobacco in their routines then potential smokers are at the largest risk of death due to smoking. Second dangerous position is of temporary quitters.

\section{CONCLUSION}

In this paper a nonlinear system of differential equations is being solved numerically using Perturbation-Iteration Algorithm. Parameters chosen from the original work indicates the situation where $R_{1}>1$.

Which means that potential smokers $P(t)$ after sometime will reach the global equilibrium point whereas occasional smokers at first will increase and then slowly will reach its estimated equilibrium point. Other smokers and quitters will initially increase or decrease but then after sometime they will attain their equilibrium. Therefore $R_{1}>1$ implies that the system has a global equilibrium, analyzed by stability analysis in [14]. In this work the observations correlate with the results provided in [14].

\section{CONFLICT OF INTERESTS}

The authors declare that there is no conflict of interests regarding the publication of this paper.

\section{ACKNOWLEDGMENT}

We thank the reviewers for their thorough efforts in editing our paper and highly appreciate the comments and constructive criticism that significantly contributed in improving the quality of the publication. The authors also thank Ms.Wishaal Khalid for proofreading our research paper.

\section{REFERENCES}

[1] Erturk, V.S., Zaman, G., Momani, S. (2012) A numericanalytic method for approximating a giving up smoking model containing fractional derivatives, Computer and Mathematics with Applications, 64(2), pp 3068-3074.

[2] Mohamed, S.A., Nahed, A.S. (2014) An analytical treatment to fractional gas dynamics equation, Applied and Computational Mathematics, 3(6), pp 323-329.

[3] Singh, J., Kumar, D., Kilicman, A. (2014) Homotopy perturbation method for fractional gas dynamics equation using sumudu transform, Abstract and Applied Analysis, 2014, ID 934060, 8 pages.

[4] Mohamed, S.A. (2015) New iterative Method for fractional Gas Dynamics and coupled Burger's equation, The Scientific World Journal, 2015, ID 153124, 8 pages.

[5] Khalid, M., Sultana, M., Zaidi, F., Khan, F.S. (2015) Numerical Solution for SIR Model of Dengue Fever, International Journal of Computer Applications, 118(21), 4 pages.

[6] Khalid, M., Sultana, M., Zaidi, F., Khan, F.S. (2015) Solving Polluted Lakes System by Using Perturbation Iteration Method, International Journal of Computer Applications, 114(4), 7 pages.

[7] Khalid, M., Sultana, M., Zaidi, F., Khan, F.S. (2015) A numerical Solution o a Model for HIV Infection CD4+ T-cell, International Journal of Innovation and Scientific Research, 16(1), pp 79-85.

[8] Zaman, G. (2011) Optimal campaign in the smoking dynamics, Computational and Mathematical Methods in Medicine, 2011, ID 163834, 9 pages.

[9] Zaman, G. (2011) Qualitative behavior of giving up smoking models, Bulletin of the Malaysian Mathematical Sciences Socitey, 34(2), pp 403-415.

[10] Lubin, J.L., Caporaso, N.E. (2006) Cigarette smoking and lung cancer: modeling total exposure and intensity, Cancer Epidemiology, Biomarkers and Prevention, 15(3), pp 517523.

[11] Garsow, C.C., Salivia, G.J., Herrera, A.R. (2000) Mathematical Models for the Dynamics of Tobacoo use, recovery and relapse, Technical Report Series BU-1505-M, Cornell University, UK.

[12] Sharomi, O., Gumel, A.B. (2008) Curtailing smoking dynamics: a mathematical modeling approach, Applied Mathematics and Computation, 195(2), pp 475-499.

[13] Zeb, A., Chohan, I., Zaman, G. (2012) The Homotopy Analysis method for Approximating of Giving Up Smoking Model in Fractional Order, Applied Mathematics, 3, pp 914-919.

[14] Alkhudhari, Z., Al-Sheikh, S., Al-Tuwairqi, S. (2014) Global dynamics of a mathematical model on smoking, Applied Mathematics, 2014, ID 847075, pp 914-919. 